Correspondence

\title{
Carotid artery disease and neurocognitive impairment
}

\author{
Thomas H. Ottens *, Diederik van Dijk
}

University Medical Center Utrecht, Department of Anesthesiology, Intensive Care and Emergency Medicine, Utrecht, The Netherlands

\section{A R T I C L E I N F O}

\section{Article history:}

Received 1 April 2017

Accepted 3 April 2017

Dear Dr. Eyuboglu,

We appreciate your well-advised comments on our article "Brain volume and cognitive function in patients with revascularized coronary artery disease" [1]. We agree with your statement that atherosclerosis should be regarded as a systemic disease that manifests in multiple vascular beds. The patients that we reported on in the study initially underwent coronary artery revascularization. They did not undergo structural screening for carotid artery disease. However, many patients in the coronary artery disease (CAD) group had risk factors for carotid artery disease such as diabetes, hypertension, hypercholesterolemia and smoking. It is indeed possible that the association that we found between CAD and smaller white matter volume is the result of generalized atherosclerosis, which can also affect the carotid arteries.

Patients who participated in our study have not received specific interventions to treat or prevent cognitive dysfunction.

Sincerely,

Thomas H. Ottens, MD, PhD

Diederik van Dijk, MD, PhD

University Medical Center Utrecht

Department of Anesthesiology, Intensive Care and Emergency Medicine

Utrecht, The Netherlands

\section{Reference}

[1] T.H. Ottens, J. Hendrikse, H.M. Nathoe, G.-J. Biessels, D. Van Dijk, Brain volume and cognitive function in patients with revascularized coronary artery disease, Int. J. Cardiol. 230 (2017) 80-84

\footnotetext{
* Corresponding author.

E-mail address: t.h.ottens@umcutrecht.nl (T.H. Ottens).
} 